\title{
The MIR properties of red QSOs
}

\author{
An-Li Tsai* \\ Institute of Astronomy, National Central University, Taiwan \\ E-mail: altsaidastro.ncu.edu.tw

\section{Chorng-Yuan Hwang} \\ Institute of Astronomy, National Central University, Taiwan \\ E-mail: hwangcvdastro.ncu.edu.tw
}

\begin{abstract}
The nature of optical/IR red color QSOs is considered to be reddened by dust with two popular scenarios, (I) QSOs are observed through the orientation of the dusty torus around the central AGN, or (II) QSOs are associated with nuclear star formation. The best wavelengths for studying the obscuration process in QSOs are IR to radio, because they are less affected by dust extinction. Our research investigates the Mid-Infrared (MIR) properties of a sample of red QSOs by using the Wide-Field Infrared Survey Explorer (WISE) All-Sky Source Catalog. These red QSOs are selected base on the QSO color excess defined by the SDSS Quasar Catalog, the latter which has overlap in sky coverage with the VLA FIRST radio survey catalog. The results from the WISE data shows that the red QSOs have significantly larger MIR emission than the normal ones have, no matter they are radio-loud QSOs (RLQs) or radio-quiet QSOs (RQQs).
\end{abstract}

Nuclei of Seyfert galaxies and QSOS - Central engine \& conditions of star formation November 6-8, 2012

Max-Planck-Insitut für Radioastronomie (MPIfR), Bonn, Germany

\footnotetext{
*Speaker.
} 


\section{Introduction}

Based on the unified model of Active Galactic Nuclei (AGN), we can observe different types of AGNs based on the line-of sight orientation of AGNs [四]. The Quasi-Stellar Objects (QSOs) are one of the most powerful type of AGNs. The typical QSOs usually show blue optical-to-ultraviolet spectral energy distributions. However, the recent observations discovered a population of red

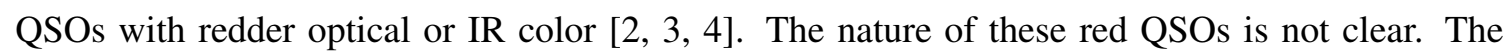
redness might be caused by obscuration either due to the dusty torus surrounding the accretion disc or dust associated with nuclear star formation in the early stages of AGN evolution. The best wavelengths to distinguish two obscuration effects are IR and radio wavebands, which are not affected by the dust extinction and can decouple different obscuration effects.

\section{Data and Selection Criteria}

\subsection{Optical Data}

So far there is no generally accepted definition of red QSOs. We define the red QSOs based on the QSO differential color [ [1], 团], $\delta(g-i)=(g-i)_{\mathrm{QSO}}-<(g-i)>_{\mathrm{z}}$, where $g$ and $i$ are SDSS $g$-band and $i$-band magnitudes, $(g-i)_{\mathrm{QSO}}$ is the QSO color, and $\langle(g-i)\rangle_{\mathrm{z}}$ is the modal value of this color at the redshift of the QSO with a redshift bin-width of 0.01 . In other words, $\delta(g-i)$ is the offset in magnitudes of the QSO $(g-i)$ from the mode value. The differential color at zero is the normal QSOs. The distribution shows a "red"tail [ [ $],$, [1, 目] at $\delta(g-i)>0.3$ but no blue counterpart at $\delta(g-i)<-0.3$. Therefore, we defined the QSOs with $\delta(g-i)>0.3$ as the red QSOs, and $-0.05<\delta(g-i)<0.05$ as the normal QSOs.

\subsection{Redshift Range}

We choose the redshift range between 0.6 and 2.0. The lower limit of 0.6 is to prevent the contamination from host galaxies, and the upper limit of 2.0 is to prevent the $\operatorname{Ly} \alpha$ emission line redshifted into optical bands.

\subsection{Radio Selection}

The SDSS-selected QSOs have some overlaps in the sky coverage of the VLA FIRST Radio Survey. We match the SDSS QSOs with the FIRST sources to study the radio properties of the QSOs. We defined the Radio-Loud QSOs (RLQs) and the Radio-Quiet QSOs (RQQs) using the Radio-to-optical ratio, $R_{\mathrm{g}}=\log \left(F_{\text {radio }} / F_{\text {optical }}\right)=0.4(g-t)$, where $t=-2.5 \times \log \left(F_{\text {int }} / 3631 \mathrm{Jy}\right)$ is the $\mathrm{AB}$ radio magnitude, and $F_{\text {int }}$ is the FIRST integrated flux density. We defined $R_{\mathrm{g}}>1$ as the RLQ, and $R_{\mathrm{g}} \leqslant 1$ as the RQQ.

\subsection{MIR Data}

We look for the MIR counterparts of the SDSS-selected QSOs within the FIRST sky coverage using the Wide-Field Infrared Survey Explorer (WISE) All-Sky Source Catalog. There are four WISE bands, 3.4, 4.6, 12, and $22 \mu \mathrm{m}$. The number of the WISE-selected QSOs is 63,128. The RLQs have more red QSOs than the RQQs have, and the red QSOs have more RLQs than non-red QSOs have. 


\section{Results and Discussion}

We compare color difference between optical, radio, and MIR, of normal and red QSOs to investigate the cause of redness.

\subsection{Optical-MIR Colors}

Optical-MIR colors are (SDSS $g$-band)-(WISE band) of the red and the normal RLQs. The red color indicates the red RLQs, and the blue color indicates the normal RLQs. The result shows that the red RLQs have larger Optical-MIR colors than the normal RLQs. Optical-MIR color comparison between the RLQs and RQQs for the red and the normal QSOs. The color difference between RLQs and RQQs in both red and normal QSOs is not as large as the difference between the red and the normal QSOs.

\subsection{MIR-Radio Colors}

MIR-Radio colors are (WISE band)-(VLA 1.4GHz) of the red and the normal RLQs. The result shows that the red RLQs have smaller MIR-Radio colors than the normal RLQs, i.e., the red RLQs have brighter MIR emission than the RQQs.

\subsection{Interpretation of Red QSOs}

The majority of the red QSOs discovered were considered to be reddened by dust. The origins could be obscured by (I) the dusty tours of the AGN or (II) the dust associated with star formation [四]. For case I, we should see the radio and the MIR properties of the red QSOs similar to those of the normal QSOs. However, there is more MIR emission in the red QSOs than in the normal ones, indicating that there is additional dust in the red QSOs. We conclude that the red QSOs can not be explained by a normal QSO obscured by the torus. The dust could be associated with star formation as suggested by case II, or the red QSO has a larger hot torus than the normal one has.

\section{References}

[1] C. M. Urry and P. Padovani, Unified Schemes for Radio-Loud Active Galactic Nuclei, PASP 107803 [astro-ph/9506063]

[2] R. L. Webster, P. J. Francis, B. A. Petersont, M. J. Drinkwater and F. J. Masci, Evidence for a large undetected population of dust-reddened quasars, Nature 375 (6531) 469

[3] G. T. Richards, P. B. Hall, D. E. Vanden Berk, et al., Red and Reddened Quasars in the Sloan Digital Sky Survey, AJ 126 (3) 1131 [astro-ph/0305305]

[4] E. Glikman, D. J. Helfand, R. L. White, R. H. Becker, M. D. Gregg and M. Lacy, The FIRST-2MASS Red Quasar Survey, ApJ 667 (2) 673 [astro-ph/0706.3222]

[5] D. P. Schneider, P. B. Hall, G. T. Richards, et al., The Sloan Digital Sky Survey Quasar Catalog. IV. Fifth Data Release, AJ 134 (1) 102 [astro-ph/0704.0806]

[6] D. P. Schneider, G. T. Richards, P. B. Hall, et al., The Sloan Digital Sky Survey Quasar Catalog. V. Seventh Data Release, AJ 139 (6) 2360 [astro-ph/1004.1167]

[7] G. Canalizo, A. Stockton, M. S. Brotherton and M. Lacy, Star formation in QSO host galaxies, New Astro. Rev. 50 (9-10) 650 [astro-ph/0603218] 